\title{
Object Tracking using Supervised Level_Set Model (SLSM)
}

\author{
V. Surendra Reddy, T. Rajyalakshmi, D. Rajya Lakshmi
}

\begin{abstract}
Conceptual-An item discovery framework discovers objects in this present reality in an advanced picture or video, in which the article can have a place with any of articles to be specific people, vehicles, and so on. So as to distinguish an article in a picture or video the frameworkneeds couple of parts so as to finish the errand of recognizing an item, an element finder, a theorem and theorem checker.In this work survey of different strategies which are utilized to distinguish an article, limit an item, order an item, extricate highlights, appearance data in pictures and recordings. The remarks are dependent on the considered writing and major problems are likewise recognized significant to the item location. A thought regarding the conceivable answer for multiple class_object identification is likewise exhibited. This work is appropriate for specialists who are learners in this area.. We initially portray the proposed system of two-stage supervised level set model in target following, at that point give summed up multi-stage adaptation for managing multiple-target . Positive decline is utilized to modify the learning after some time, empowering following to proceed under fractional and add up to impediment. Test results in various testing arrangements approve the viability inproposed strategy.
\end{abstract}

\section{PROLOGUE:}

Article following, which alludes to assignment of producing the directions of the moving items in an arrangement of pictures, is a testing research subject in area of PC vision. The issue and its trouble rely upon a few components, for example, the measure of earlier information about the objective article and sort of parameters followed example area, scale, point by point form. In spite of the fact that there is some accomplishment by fabricating trackers for explicit item classes, following conventional genuine articles has stayed testing because of precarious lighting_condition, present varieties, see point manipulation, and camera commotion and so forth. Early following strategies [1], [2] utilize settled a model to portray the objective, which can't effectively follow the objective over prolonged stretch of time.To beat this disadvantage, some following calculations endeavour to refresh the objective appearance after some time inonline way. The appearance_models received by these strategies incorporate histogram [3], subspace_models [4] and in addition inadequate portrayal models [5], [6]. Additionally, a few scientists resort to receiving critical learning strategies to made trackers simple to recognize the objective from its experience. The techniques dependent in boosting [7], [8]

Revised Manuscript Received on August 19, 2019.

V.SurendraReddy,

Professor,

Newton'sInstituteofScience\&Technology,AndhraPradesh,India

T.Rajyalakshmi,.Assistant.Professor,

UniversityCollegeofNarasaraopet(JNTUN),A.P,India

D.Rajya Lakshmi, Professor, University College of Narasaraopet (JNTUN), AndhraPradesh,India. and svms [9], [10] demonstrate great execution and pull in much consideration. Conversely with building two different models for the objective and foundation individually, learning methodologies are increasingly disposed to seize more segregation between them. Notwithstanding having the promising execution, these conventional trackers confront a down to earth issue that they utilize oval to surmise the followed target.In any case, protests by and by have complex structures which can't be all around depicted by basic geometric structures, in Figure. 1(a) for a few precedents. Since the square shape box utilized for exhibiting the followed target specifically decides the examples to be separated in resulting target appearance displaying, it is basic factor to following execution. Wrong target introduction effortlessly results in execution misfortune because of the contamination in non-object locales dwelling inside square shape box. So as to more readily fit the item structure, a few techniques receive the scale choice component that means to scan for better scale which covers objective precisely. An instinctive thought was to run calculation in various scales, at that point select the one amplifying the article capacity of the following calculation. This choice instrument is likewise reached out to introduction. At the same time controlling scale and introduction, the measurement predisposition for objective conveyance, in Figure.1(a), to some degree, improves target portrayal and following estimation. By and by, all these introduction alterations are as yet dependent on basic geometric structures, (for example, square shape and oval), which unavoidably present countless pixels when utilized in giving certifiable article different structures. Preferably, a superior way to portray the objective is to utilize the precise form along with objective's surface. A few endeavours in the writing is made to utilize outline or form, dividing procedure for dynamic following [11], [12]. Interestingly with unequivocal portrayal of shapes in parametric dynamic forms, for example, wind display [13], level_set method [14]- [17] is an understood portrayal of shapes and ready to manage changes. The essential thought of the dimension set methodology is to insert the shape as the zero dimension set of the diagram of higher dimensional_capacity.At that point develop the diagram with the goal that this dimension set moves as per the endorsed stream until the point when it limits a picture based vitality work. Paired dimension set_model, explained in [18], utilizes two-esteemed dimension set capacity to supplant the marked separation work utilized in customary Chan_Vese way [17]. Since it

Published By:

Blue Eyes Intelligence Engineering

\& Sciences Publication

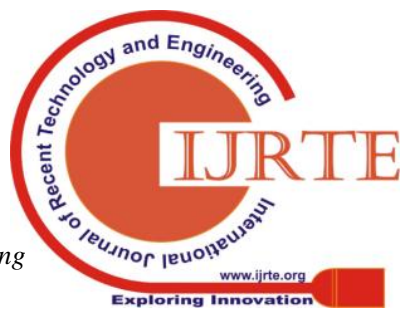


maintains a strategic distance from the re-instated procedure of the dimension set capacity in every cycle and additionally the awkward numerical acknowledgment, it extraordinarily enhances computational effectiveness and thus is increasingly appropriate for following assignments. All things considered, from an execution point of view, the parallel dimension set model increasingly disposed to portion out the locale with reliable power, like the limit division technique. However, as of late, a few works have been introduced to visual following, presenting the earlier target information in a dimension formalism is yet difficult, since dimension set structure goes for ideally gathering areas whose picture elements has comparative element marks.This made troublesome for some ways to deal with dependably section and track genuine world, multi-mode protests before perplexing, jumbled foundations.

We explain here administered level_model for certifiable items shape following. Instead of working with power predictable course, the bend advancement in supervised mode level set was target-arranged and directed by information of the explicit focuses in following application. Boosting_approach utilized in online development ofobjective appearance show because of its solid capacity in recognizing the objective. At that point the educated target display is fused to demonstrate the dimension set form probabilities by Bayesian_way, driving the bend meet to the hopeful locale with most extreme probability of being followed target. At long last, examples extricated from precise target area are nourished back to the boosting method.We utilize positive decline rate to change objective learning after some time, that empowers following to proceed under incomplete and add up to impediment. We right off the bat portray the proposed automate in two_stage supervised level set model in single target following, whose starter results likewise introduced in earlier meeting paper [19]. At that point we oddity propose the summed up multistage supervised level set model in managing multiple targets. Figure.1(b-g) demonstrates following instances of our strategy in different testing cases.

\section{WRITING SURVEY:}

$>$ In complexity to now-a-days trackers that utilization bouncing_box to determine the followed target.

$>$ Support Vector Machine algorithm utilized is here.

$>$ In regular dimension models just stresses the territorial power consistency and don't think aboutpriors.

$>$ Unfit to follow effectively quite a while.

$>$ They continued with online procedure, histogram techniques and so forth.,.

\section{PROPOSED TECHNIQUE \& RESULTS}

We represent an administered model for certifiable articles form following. Instead acting towards force reliable bearing, the bend development of supervised level set model is goal-situated and regulated by information of explicit focuses in following usage. Boosting method was utilized in online development of objective appearance show because of its solid capacity in recognizing objective from experience. At that point the educated target show is

consolidated to display the dimension set form by Bayesian_way, driving the bend meet to applicant district with greatest probability for being followed target. At long last, examples extricated from exact target area are encouraged back to this technique. We utilize decline rate in modifying the objective learning after some time, that empowers following in proceeding under halfway and add up to impediment. We initially portray the motorize of twostage SLSM in single following, in which starter results are additionally displayed in the earlier paper [19]. At that point we oddity proposed summed up multiple-stage SLSM in managing multiple-target following. Figure.1(b-g) demonstrates following instances in our strategy in different testing cases.

Stage_1: Utilize the calculation called SLSM.

Stage_2: This strategy extricates precise shapes of objective as following item.

Stage_3: Accomplishes better depiction of non-inflexible item which decreases foundation contamination.

Stage_4: SLSM could guarantee progressively precise assembly in correct item of following applications.

Stage_5: This strategy has been actualized on single target succession utilizing two stage SLSM technique.

Stage_6: Setting of following, we defined the bend advancement in SLSM by explicit information of objectives we need in tracking, that was studied in web based boosting way.

Stage_7: Interestingly with regular power consistencydimensionmethods, our method was object based and can increasingly precise assembly to correct focuses in following applications.

Simulation Analysis:

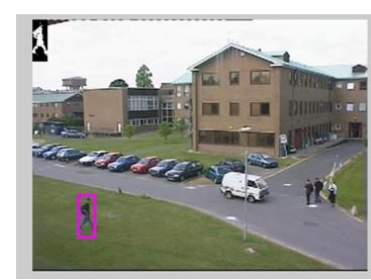

(a) Building video frame

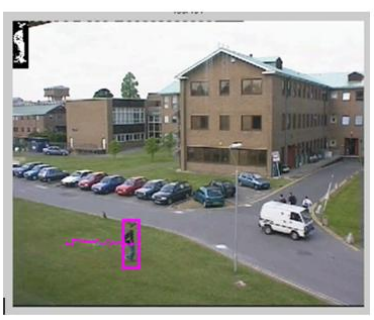

(c) video frame 125

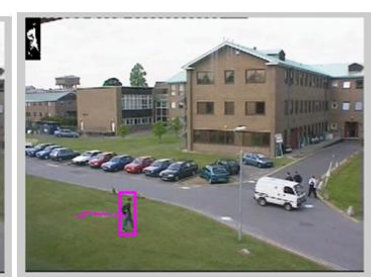

(b) video frame 23

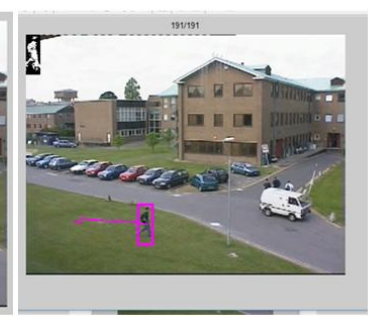

(d) video frame
191 


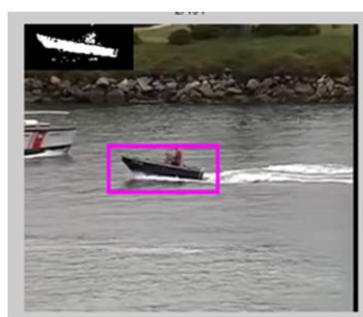

(a) Cost guard video frame

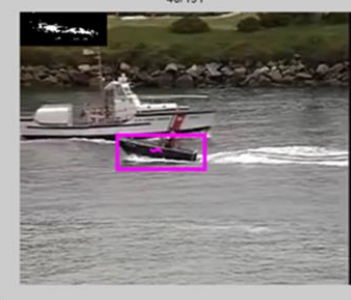

(c) video frame 125

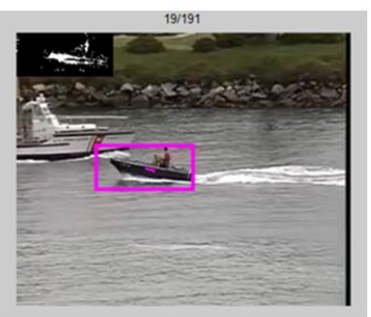

(b) video frame 23

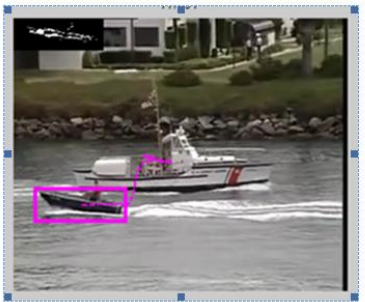

(d) video frame 191

\section{CONCLUSION:}

We introduced a new regulated dimension set_ model in our work in non-unbending articles shape following. By setting of following, we redefined the bend development in supervised level set model by explicit information about objectives we need in following, that is found out in a web based boosting way. Thus, interestingly with traditional power based dimension set techniques, this methodology was object-arranged and progressively precise assembly to correct focuses for following applications. We right off the bat proposed the automate in two stage supervisedlevel set model in one goal following, at that point proposed summed up multiple_stage supervised level set model in managing multiple_targets. Test results in various testing video arrangements have confirmed that this strategy is viable in many muddle scenes.

\section{REFERENCES}

1. A. Elgammal, R. Duraiswami, and L. S. Davis, "Probabilistic tracking in joint feature-spatial spaces," in Proc. IEEE Comput. Soc. Conf. CVPR, Jun. 2003, pp. I-781-I-788.

2. P. Pérez, C. Hue, J. Vermaak, and M. Gangnet, "Color-based probabilistic tracking," in Proc. 7th ECCV, 2002, pp. 661675.

3. D. Comaniciu, V. Ramesh, and P. Meer, "Kernel-based object tracking," IEEE Trans. Pattern Anal. Mach. Intell., vol. 25, no. 5, pp. 564-577, May 2003.

4. D. Y. Kim and M. Jeon, "Spatio-temporal auxiliary particle filtering with 1-norm-based appearance model learning for robust visual tracking," IEEE Trans. Image Process., vol. 22, no. 2, pp. 511-522, Feb. 2013.

5. X. Mei and H. Ling, "Robust visual tracking and vehicle classification via sparse representation," IEEE Trans. Pattern Anal. Mach. Intell., vol. 33, no. 11, pp. 2259-2272, Nov. 2011.

6. S. Zhang, H. Yao, X. Sun, and X. Lu, "Sparse coding based visual tracking: Review and experimental comparison," Pattern Recognit., vol. 46, no. 7, pp. 1772-1788, 2013.

7. S. Avidan, "Ensemble tracking," IEEE Trans. Pattern Anal. Mach. Intell., vol. 29, no. 2, pp. 261-271, Feb. 2007.

8. H. Grabner, C. Leistner, and H. Bischof, "Semi-supervised on-line boosting for robust tracking," in Proc. ECCV, 2008, pp. 234-247.
9. S. Hare, A. Saffari, and P. H. S. Torr, "Struck: Structured output tracking with kernels," in Proc. IEEE ICCV, Nov. 2011, pp. 263-270.

10. L. Zhang and L. van der Maaten, "Structure preserving object tracking," in Proc. IEEE Conf. CVPR, Jun. 2013, pp. 18381845 .

11. L. Lu and G. D. Hager, "A nonparametric treatment for location/segmentation based visual tracking," in Proc. IEEE Conf. CVPR, Jun. 2007, pp. 1-8.

12. Z. Cai, L. Wen, Z. Lei, N. Vasconcelos, and S. Z. Li, "Robust deformable and occluded object tracking with dynamic graph," IEEE Trans. Image Process., vol. 23, no. 12, pp. 5497-5509, Dec. 2014. 